\title{
Las organizaciones, las comunicaciones y el cambio
}

Víctor L. TORRES TEJADA

Es una tarea dificil el estudiar conceptos que son profusamente discutidos, rara vez confrontados y sobre los que se concluye poco. El cambio organizacional es uno de ellos. No sólo es complicado precisarlo, sino que a veces lleva a los entendidos a sostener posiciones extremas sobre su significado.

Los autores que se dedican al estudio de este campo manejan perspectivas analiticas bastante disimiles: están los que examinan los mecanismos y formas del inicio de los cambios, y los que evalúan y planifican los cambios futuros, además de quienes intentan explicar los fundamentos filosóficos, sociales, económicos y psicológicos del fenómeno. Y así sucesivamente.

En más de un caso los conceptos quedan en la imprecisión o en el ámbito particular que les fija cada expositor. Sólo excepcionalmente se estudia la problemática del cambio organizacional considerando la óptica (de)formativa que diversos autores defienden y, por consiguiente, privilegia algunos aspectos descuidando otros, y los estudios sobre la materia resultan segmentarios y/o notoriamente sesgados. No podemos asegurar que el presente artículo aclarará las incertidumbres señaladas, pero se hará un esfuerzo por clarificar algunos conceptos y, sobre todo, se intentará delimitar el rol que las comunicaciones desempeñan en el cambio organizacional.

Debemos partir recalcando que la premisa básica de los estudios realizados en este campo, y que suscribimos en este trabajo, establece que para sobrevivir las organizaciones re- 
1. Ogburn, William. Recent Social Trends. Nueva York: McGraw Hill Inc., 1993.

2. Hodgetts, Richard [y] Steven Altman. Comportamiento en las organizaciones. México: Editorial Interamericana, 1981. quieren resolver favorablemente la dicotomía existente, de no muy fácil solución, entre la continuidad y el cambio; el antagonismo entre la transmisión de patrones culturales que buscan afianzarse, y la introducción de elementos nuevos dirigidos a posibilitar modificaciones. Estos nuevos factores deben propiciar la ruptura con lo anterior, con "lo tradicional", pero a su vez deben generar mecanismos que aseguren su propia consolidación.

Al respecto, unos opinarán que sólo se trata de frases declarativas, otros de descripciones teóricas sobre situaciones ideales, no faltarán quienes enfatizarán la dificultad de introducir el cambio en sus organizaciones y habrá quienes, por el contrario, solicitarán recetas para su puesta en marcha.

Debemos reconocer que en las organizaciones se dan procesos sociales de marcada continuidad, a los que lamentablemente no se les ha dado la importancia debida. Sólo a partir de su conocimiento es posible iniciar la presentación e incorporación del cambio como alternativa de vida institucional. Para lograr éxito en este propósito, el sistema de comunicación resulta ser un extraordinario mecanismo de apoyo y de evaluación.

Parece que conceptos tales como 'modernidad', 'liberalidad' y 'competitividad' traen como novedad la idea del cambio organizacional y social. Sus difusores aconsejan prepararnos para estos nuevos tiempos de fuerte competencia y de profundos cambios. Sin embargo, es de justicia reconocer que el concepto de cambio social con sus repercusiones siempre ha preocupado a los interesados en el análisis riguroso de la marcha de las organizaciones. En general, el tema ha sido abordado constantemente por todos los estudiosos de los sistemas sociales. Así, por ejemplo, al inicio de la década de los años 30, William Ogburn ${ }^{1}$, en su libro Recent Social Trends, considerado como uno de los precursores de lo que hoy conocemos como la Teoría de Sistemas, sustentaba con abundante material probatorio la influencia recíproca que se da entre el medio ambiente y las organizaciones, y especialmente con respecto al impacto que ocasionan las nuevas tecnologías al traducirse en un ámbito laboral específico. Aunque estos estudios son parciales, testimonian que siempre ha estado presente la preocupación por el estudio del cambio y de la innovación en las organizaciones.

Autores como Hodgetts y Altman ${ }^{2}$ se interesan por los orígenes del cambio y por la forma en que los agentes y los participantes deben prepararse para detectarlo o iniciarlo y 
observan que el cambio empieza cuando algo en la organización no sincroniza con los tiempos. Kolasa se interesa por el proceso de asimilación del cambio, por el aprendizaje y las enseñanzas que éste deja. Robert Gilbreath ${ }^{3}$, por su parte, incidirá en la temporalidad del cambio y su medición como una variable importante a considerar, especialmente si se desea evaluar el impacto y si se privilegia el efecto del cambio. Asimismo, Robert Blake 4 y Anni Bartoli ${ }^{5}$ demandan conocer el tipo de cambio al que se alude, su dirección e intensidad, así como la intencionalidad con la que buscamos que éste se dé en las organizaciones; de este modo, la lista puede continuar hasta escribir un tratado sobre las formas y estilos en la que los autores enfocan este tema.

Un aspecto interesante de rescatar, al revisar la literatura, es el reconocimiento de que el concepto cambio organizacional no posee más la carga negativa o la connotación peyorativa de la que estaba impregnada, mucho menos se teme nombrarlo. Ahora su aceptación tiene menos resistencia y, en no pocos casos, se reconoce lo positivo que puede ser si se le sabe manejar con propiedad. Incluso se afirma que requiere ser usado como impulsor de innovaciones en beneficio de las instituciones y como creador de situaciones que pueden contribuir a la marcha de las organizaciones y a la resolución de conflictos internos.

Otro punto de acuerdo, un tanto difuso, se da en el señalamiento de que el cambio organizacional es todo aquello que se nota diferente a lo existente previamente y que puede ser reconocido, con claridad, como algo nuevo. Esta aproximación al concepto tiene la gran ventaja de congregar a más de un estudioso del tema, comprendiendo desde las simples modificaciones en los procedimientos de las empresas, que no necesitan de una mayor transformación de las personas, hasta el cambio en las formas de gestión y la propiedad empresarial que implica toda una nueva concepción de la dirección y el manejo empresarial, pasando por las innovaciones tecnológicas que requieren una adecuación a los tiempos.

Un punto de menor acercamiento se da cuando se indica que el concepto de cambio en las organizaciones implica mucho más que la simple idea de mejorar, de lograr mayores beneficios o de realizar algo novedoso; sin duda estos conceptos están inmersos en la idea de cambio organizacional, pero éste es más que la sumatoria de todos ellos.

Es posible notar, sin dificultad, que sustantivos como innovación, transformación, evolución, renovación, revolución y otros se usan de manera intercambiable con el con-
3. Gilbreath, Robert. La estrategia del cambio. Bogotá: McGraw Hill, 1989.

4. Blake, Robert. La estrategia para el cambio organizacional. Delaware: Addison Wesley Iberoamericana, 1991.

5. Bartoli, Annie. Comunicación y organización. La organización comunicante y la comunicación organizada. Barcelona: Paidós, 1992, p.177. 
6. Bartlett, Alton y Thomas Kayser. Cambios de la conducta organizacional. México: Trillas, 1980, p. 88. cepto cambio; depende de a quién se lea y qué se desea enfatizar para cruzarse con la variedad de términos indicados. Algunos autores los usan como sinónimos simplemente para no repetirlos y ello complica aún más su conceptualización. Desde una perspectiva de significación cada término tiene su propio ámbito; unos con más fuerza que otros explican lo que sucede en las empresas y en general en la sociedad, cuando algo es diferente de lo que fue antes. Bartlett y Kayser señalan que aparentemente hay un acuerdo para estar en desacuerdo sobre el concepto cambio organizacional y lo que con él se desea connotar. Estos autores van más allá en sus críticas e indica que:

\begin{abstract}
“...las teorias del cambio tienden a explicar las interacciones dinámicas de un sistema, sin proporcionar clave alguna para identificar las acciones de palanca estratégicas que permitan realizar dichos cambios. Estas teorias son adecuadas para quienes quieren observar el cambio social, pero son de poca ayuda para quienes lo practican; son teorías del cambio y no del cambiar"6.
\end{abstract}

Sin pretender evadir la rigurosidad conceptual, creemos que nuestro compromiso se sitúa también en señalar los momentos y los procesos para incorporar el cambio en las empresas. Si la definición del concepto es compleja, queda esperar que su operatividad ayude a su mejor comprensión.

Cuando el entorno de la organización era relativamente estable, el cambio fue un proceso sencillo ante el cual las personas que manejaban las organizaciones reaccionaban y tomaban, según las circunstancias, las medidas pertinentes para adecuarse a los tiempos. Esta situación ha variado por completo en razón de la forma en que el desarrollo de la humanidad ha ido modificándose. El ritmo de vida de las sociedades modernas nos ha permitido conocer avances significativos en las esferas culturales, científicas, tecnológicas, sociales, administrativas, etc., los que representan cambios que se constituyen en fuerzas dinamizadoras en el entorno y que no pueden ser ignoradas por quienes dirigen las instituciones. En este sentido, una organización que quiere permanecer en el medio y operar con los tiempos deberá ser consciente de que el cambio es en la actualidad una actividad necesaria y factible de ser planificada, al igual que las estrategias y politicas institucionales, dando ello sentido y permanencia a las organizaciones y permitiéndoles su prospección. 
Los estudiosos de este campo, indica Marvin Olsen, han considerado que:

“...las fuentes del cambio pueden ser divididas en dos grandes categorias, a) las externas a la organización que producen presiones (estímulos); y, b) las internas a la organización que producen tensiones (conflictos). Al analizar las causas externas se señalan cuatro factores como los influyentes en los cambios: el medio ambiente, la población en general, los individuos en particular y las nuevas tecnologias; en el aspecto interno se indican tres factores: los cambios de y en los integrantes, la variación en la cultura organizacional; $y$, las funciones y/o disfunciones de las organizaciones que dan origen a conflictos"7.

Los preocupados por esta área de estudio conocemos que la Teoría de Sistemas recoge estos postulados y les da mayor amplitud y precisión, enfatizando la interrelación entre medio ambiente y organizaciones, e incidiendo de manera más específica en sus mutuas afectaciones.

Esta nueva concepción del cambio organizacional es objeto de múltiples análisis por los autores modernos quienes, desde diferentes perspectivas, enfatizan su significado filosófico, su base científica o sus implicaciones sociales; en otras ocasiones analizan sus repercusiones a nivel de los individuos y de las organizaciones (especialmente de las empresas). Robert Gilbreath, autor que ha mostrado interés y preocupación por la aplicación del cambio en las empresas, en su libro La estrategia del cambio anota que:

“...cuanto mayor conocimiento intimo tengamos del cambio y sus fases, más fácilmente podemos salir adelante en un mundo dominado por su presencia. Debemos entender y confrontar al cambio para conquistarlo, igualmente debemos entender nuestros límites para explotarlo, y capitalizar las nuevas formas empresariales que el cambio genera en beneficio de cualquier innovación a favor de la nuestra"8.

Las fases o etapas a las que hace referencia Gilbreath, las desarrollaremos con mayor amplitud cuando abordemos la planificación del cambio y la forma de incorporarlo a las organizaciones.

Es necesario, por lo tanto, planificar el cambio y anticipar sus posibles resultados, especialmente cuando deseamos innovar nuestras organizaciones; también es factible monitorear el cambio y, a veces, propiciarlo e incorporarlo, bus-
7. Olsen, Marvin.

The Process of Social Organization.

Indiana: Indiana University Press, 1968, p. 140.
8. Gilbreath, Robert. Op. cit. p. 2. 
cando obtener los objetivos prefijados de nuestras instituciones. Del mismo modo, es posible observar en otras organizaciones los resultados que los cambios están produciendo e incorporar a nuestras empresas aquéllos que consideremos que puedan ser beneficiosos. Sin embargo, debemos indicar con claridad que no todo cambio debe necesariamente ser incorporado, ni favorece a todas las empresas por igual.

\section{EL CAMBIO COMO PROCESO BÁSICO: ¿CAMBIAMOS O NOS CAMBIAN?}

La sociedad no es un sistema estático, se encuentra en constante dinamismo y permanente cambio. Algunos de éstos son de evolución lenta, no perceptibles fácilmente; otros son más fáciles de identificar, especialmente cuando se trata de innovaciones tecnológicas, que dificilmente pueden ignorarse, pues afectan la marcha de la colectividad. Es importante que los seres humanos aprendan a reconocer y a manejar el cambio; pero, es más trascendente aún que sepan producir cambios. Este principio de dinamismo constante, presente en los sistemas sociales y grandes agregados, es válido también para estructuras pequeñas, instituciones y grupos, y con mucha más razón para los individuos que son los generadores de los cambios. La inmanencia del cambio debe ser estudiada con detenimiento, en cuanto es parte de la vida de los seres humanos que forman los grupos y organizaciones, y, en tanto, son ellos quienes lo generan, quienes tienen que adecuarse a él, aceptarlo, adaptarlo y asumirlo. Es a partir de esta nueva situación que se podrán generar nuevos cambios.

Sin ánimo de ahondar en las discrepancias, no podemos dejar de señalar la existencia de una polémica presente entre los estudiosos del cambio organizacional. Algunos autores enfatizan la necesidad de buscar primero el cambio en los individuos, sus actitudes, sus hábitos y como consecuencia de dichos cambios, se cambiará la organización. Se parte de considerar ésta como un ente constituido por la suma de individuos; luego se infiere que se puede renovar la organización cambiando la forma de actuar de sus miembros. Uno de los más claros ejemplos del énfasis en esta dirección es el entrenamiento individualista frente al cual Katz y Khan señalan que la debilidad de este enunciado está dada por la “... simplificación extrema en la relación causal que se establece, pasando por alto las interrelaciones de las gentes en una estructura, y, además, que todo se encuentra 
dentro de un sistema que los afecta y del cual los individuos son una de las partes" 9 .

La misma critica hacen Luthans y Kreitner, quienes añaden que este enfoque pierde de vista el hecho de que la conducta organizacional debe considerarse como un todo, y no como un agregado de conductas individuales; éstas, además, se presentan en un contexto en el cual se inscriben las organizaciones, contexto que juega un rol importante en su actuar.

Obsérvese que las campañas motivacionales y las de identificación con los objetivos de las empresas poseen, en mucho casos, esta visión parcial del cambio organizacional, así como del rol que desempeñan los individuos que la integran. Se presume acá que el cambio de conducta y actitud de los individuos producirá necesariamente una organización totalmente diferente. A veces, esta misma critica podemos hacerla a los comunicadores que asocian linealmente cantidad de procesos comunicativos con cambios positivos en la empresa. Igual crítica la extendemos a los administradores de personal que recurren a principios conductistas, pensando beneficiar a la empresa y a los trabajadores, cuando lo que realmente quieren es modificar aspectos estructurales.

Las críticas igualmente reconocen que el intentar manejar y controlar todas las variables (internas y externas) en las organizaciones es tarea que sólo realizaría un equipo dedicado exclusivamente a este propósito. Existe consenso entre los autores, aun en los casos extremos, acerca del hecho de que el cambio tiene que iniciarse en algún momento y por algún punto. El acuerdo de las personas que integran una organización puede ser un buen indicativo del punto de inicio del cambio; sólo les piden reconocer que si se desea iniciar el cambio con las individualidades no se pierda de vista a los otros componentes, ni se busque dar prioridad a uno sobre el otro.

Otros autores indican que primero es necesario cambiar las estructuras y una vez modificadas ellas permitirán recién una adecuación de los individuos a las nuevas formas organizativas. Señalan que el cambio de estructuras es fundamental y debe ser el punto inicial; luego, las organizaciones posibilitarán la adaptación de los individuos y los prepararán para operar en ella, así como para generar los ajustes que sean necesarios. La crítica a este planteamiento radica en que supone la misma relación causal vista en la situación anterior; asume un lógico proceso deductivo, del todo a la parte, que no se da de forma tan mecánica. Implica, también, contar con individuos capacitados para operar el cambio o 
10. Schein, Edgar H. Psicología de la organización. México: Prentice Hall Inc., 1991, p. 224 por lo menos con los conocimientos necesarios para reconocerlo, aspectos que no son fáciles de darse en sistemas donde el cambio no es la práctica cotidiana.

Efectuando en este sentido un breve análisis de nuestra realidad observamos que las críticas más severas que se han hecho a la implantación de la calidad total en las empresas es haberlo hecho sin la debida preparación del personal. Pero, por otro lado, es preciso reconocer que el propio sistema no puede preparar a los individuos para una situación hipotética a darse en el futuro; sus estructuras nunca lo hubiesen permitido. Lo mismo sucede cuando hablamos de cambio a nivel societal ${ }^{10}$. El dilema se puede presentar con las siguientes preguntas: ¿Cambio organizacional expuesto o cambio impuesto? Como se comprenderá en estos planteamientos existe una variedad de componentes entremezclados, desde la concepción que se tiene del ser humano, hasta ingredientes de tipo ideológico.

La intención de este trabajo no es avalar ni inclinarse por una de las corrientes antes mencionadas. No intentamos descubrir quién es primero "si el huevo o la gallina". Más de una vez estas posiciones polares han llevado a los estudiosos a perder de vista el objetivo: entender una realidad para luego transformarla con mayores y mejores elementos de análisis. Bástenos con señalar que no hay respuesta univoca a este dilema, cada empresa manejará la implementación del cambio dependiendo de sus necesidades y del énfasis que quieran darle a uno u otro de los elementos indicados o a la posibilidad, siempre presente, de una combinación de ambas ópticas. En cualquier caso, que no sea el dogmatismo (enemigo de lo científico) el que nos ciegue y no nos permita, en primer lugar, reconocer el cambio; en segundo término, sentir la necesidad de incorporarlo, y, finalmente, aplicarlo de manera apropiada a cada realidad, usando los instrumentos necesarios y transitando los caminos adecuados en busca de su efectividad en las organizaciones. Que la búsqueda de esta meta no nos impida escoger los medios moralmente válidos con el fin de trabajar adecuadamente en las organizaciones, ni permitamos, tampoco, que las discusiones teóricas paralicen nuestro actuar.

El cambio empresarial que deseamos destacar contemplará ambas ópticas, pero enfatizará la dinámica interna. Queremos saber lo que sucede con las empresas y sus directivos cuando se les confronta con la necesidad de incorporar procesos innovadores que se originen tanto al exterior como al interior de ellas. Edgar Schein, considera que: 
"Todo proceso de cambio implica, no solamente el aprendizaje de algo nuevo, sino también el desaprendizaje de algo que ya estaba presente y probablemente bien integrado en las relaciones sociales de las personas $\mathrm{y}$ en las formas rutinarias de operar de las empresas" ${ }^{11}$.

Queremos conocer los retos que son necesarios vencer para hacer frente al cambio, sobre todo sabiendo que no es dable esperar a que una actividad realizada por mucho tiempo arroje fracasos o pérdidas y estudiar la resistencia que pueda ocasionar la incorporación de actividades innovadoras que han probado ser necesarias y que otorgan un mayor beneficio.

La resistencia al cambio y las formas de vencerla es otro de los problemas abordados cuando se toca la temática del cambio en las organizaciones. Se señala una natural resistencia de los individuos y de las instituciones a dejar de hacer lo que se acostumbraba e iniciar un proceso nuevo, donde el aprendizaje se asume como lo más costoso. Annie Bartoli nos previene de los riesgos de forzar cambios en las organizaciones no preparadas para ello. Igualmente, Robert Blake indica la necesidad de una planeación del cambio para evitar riesgos innecesarios al momento de incorporarlos en la cultura empresarial. En general, los autores tienden a explicar la resistencia al cambio como un fenómeno exclusivamente psicológico: el miedo a lo desconocido, el temor a experimentar con algo novedoso que está fuera de la rutina, o la mente cerrada de las personas para aceptar innovaciones. Sin embargo, desde la perspectiva organizacional, se deben considerar, además, los procesos sociales como la adaptación de las instituciones a las innovaciones tecnológicas, los condicionantes del orden social e institucional y los valores compartidos, así como los patrones culturales que ellos han creado y mantienen (lo que permite formas de cohesión grupal), sin olvidar que los beneficios que se pueden obtener del cambio dependen en mucho de un tácito consentimiento institucional.
11. Gilbreath,

Robert. Op. cit., p. 4. 


\section{¿PERO, DE QUÉ CAMBIO ORGANIZACIONAL HABLAMOS?}

Si alguien entiende cómo funciona algo, debe tratar de cambiarlo

KURT LEWIN

12. Gilbreath, Robert. Op. cit., p. 4.
“... el cambio en las organizaciones no se da en abstracto, no existe sin efectos; nuestra habilidad de percibir el cambio está limitada por nosotros mismos; y, nuestra forma de administrarlo está limitada por nuestra habilidad para percibirlo y aplicarlo"12.

Por ello la importancia de detectar procesos organizacionales con cierta continuidad que permitan percibir la necesidad del cambio y poseer los instrumentos adecuados para aplicarlo. Otro aspecto un tanto olvidado en estos estudios son las evaluaciones constantes que es necesario realizar para monitorear un proceso de cambios que garantice innovaciones duraderas. 
Nos interesa enfatizar estos principios pues ellos serán revisados con detenimiento en las organizaciones empresariales que estudiaremos en esta investigación. Debemos conocer este fenómeno, el cambio en profundidad con el fin de sacar el mayor beneficio al momento de implementarlo. Si podemos reconocer y propiciar los cambios, especialmente los que afectarán con cierta permanencia la marcha de nuestras organizaciones, podremos preparar a la gente para lidiar con él, o si sabemos identificar los cambios que nos serán beneficiosos podremos inducirlos, incorporarlos y administrarlos. No debemos esperar que el cambio en nuestras organizaciones sea fruto de la generación espontánea, pues en este caso habremos perdido todo control sobre éste.

En no pocos casos las empresas han tenido que reaccionar frente a los cambios, ello les hace perder control y fundamentalmente los obliga a tener una posición de desventaja frente a procesos que deben ser más bien claramente planificados. Por ello, en la literatura del cambio empresarial, se señala que las empresas están expuestas a dos tipos fundamentales de cambios:

\section{Cambios programados}

Significa prepararse para el cambio antes de que éste aparezca. Las organizaciones, como queda dicho, necesitan de individuos formados especialmente en la lectura de indicadores que les ayuden a vislumbrar la necesidad de cambio. Ello obliga a la organización a estar en constante alerta no únicamente en lo que deviene en obsoleto, sino también en lo que pueda y necesite mejorarse. Significa, también, que las empresas están convencidas de que ésta es una inversión que están dispuestas a realizar; la mejor forma de convencerlas es demostrarles que los beneficios superarán los costos.

\section{Cambios de reacción}

Significa que las fuerzas del cambio han sido claramente percibidas como entes actuantes en las organizaciones y a éstas sólo les queda reaccionar pronto frente a ella y buscar de adecuarse lo más rápidao y de la mejor forma posible. Pensamos que para las organizaciones de nuestro medio, los mecanismos de realimentación que vamos a plantear serán, fundamentalmente, cambios de respuesta frente a procesos de comunicación ya existentes pero mal 
diseñados o incompletos, y frente a los cuales sólo nos queda darles una pronta adecuación. El grado de adaptación a este tipo de cambios dependerá de la capacidad para percibir la magnitud del cambio que está operando; por ello, creemos que la propuesta de intercomunicación que se presenta deberá ser tomada en su exacta dimensión. En este cambio de reacción, al igual que en el cambio anterior, los beneficios son inmensamente superiores a los costos.

\section{PLANIFICACIÓN DEL CAMBIO EN LAS ORGANIZACIONES}

No se intenta presentar en este trabajo "recetas" o soluciones simplificadas sobre el cambio en las organizaciones, sobre todo en el ámbito empresarial de nuestro medio. Sin embargo, la penosa constatación de abundantes estudios preocupados por los aspectos descriptivos y por la presentación parcial de ciertos estados de las organizaciones, nos lleva a sistematizar mecanismos de planificación para incorporar el cambio. Esta sistematización no implica la adopción de planes de acción centralizados ni rígidos, ni en un tiempo tan breve que haga imposible evaluarlos.

Como ya se precisó, Annie Bartoli indica que los riesgos en los que incurren las organizaciones que improvisan los cambios son tan serios que acarrean innecesarias aventuras empresariales que pueden echar por tierra la inversión y poner en peligro la gestión, pero fundamentalmente hacer fracasar los intentos de cambio. Se señalan tres como los errores más comunes en los que incurren las organizaciones que desean cambiar sin una planificación adecuada:

\section{El riesgo de la prisa}

Toda gestión tendiente al cambio requiere de una "cronobiologia" (respeto por los ritmos de vida de la organización). Actuar apresuradamente puede bloquear el sistema $\mathrm{y}$ tener consecuencias contraproducentes.

\section{El riesgo de la novedad}

En general los cambios que han dado resultado en otras empresas ejercen una fuerte atracción en los hombres de empresa que buscan lo operativo, lo concreto, lo que dé frutos. Sabemos que ninguna solución preestablecida puede 
resolver problemas específicos; los principios rectores y los ejes metodológicos sí pueden adaptarse a diferentes situaciones.

\section{El riesgo de la desadaptación}

Otro error común parte de creer que los procesos de reorganización resuelven los problemas de las empresas; las reorganizaciones no son las panaceas que salvan todas las problemáticas, pueden generar expectativas imposibles de cumplir; es más, en lo mediato no resuelven los problemas, los agravan.

El costo de oportunidad debe ser otro criterio a considerar en este primer momento de identificación de recursos y de expectativas generadas en el personal, especialmente si se ha logrado su compromiso. Dejar pasar la oportunidad de cambiar puede ser a la larga resultar más dificil y sin duda mucho más oneroso de lo que fue al momento de identificar la relación existente entre problema, solución, recursos y compromisos. El costo de oportunidad debe ser evaluado con mucha objetividad en las empresas con el fin de no precipitar el cambio, pero tampoco retrasarlo innecesariamente. Este concepto, tan grato a los inversionistas, debería ser un elemento importante a la hora de manejar e implementar el concepto cambio en las empresas.

Por lo expuesto queda clara la necesidad de elaborar una estrategia que nos ayude a identificar el cambio, a incorporarlo y asumirlo, pero también debe resaltarse que cualquier estrategia que se adopte no debe constituirse en un modelo cerrado que impida su adaptación ni que anule las capacidades creativas. Igualmente, debemos enfatizar el hecho de que ninguna estrategia para introducir cambios en las organizaciones tendrá éxito si no se cuenta con una decisión política en los más altos niveles de la empresa, que manifiesten su apoyo a dicho proceso, y tampoco será factible de implementar si no se generan mecanismos comunicacionales y participativos que nos ayuden a evaluar el avance del proceso. El apoyo en la evaluación implica, a su vez, otro tipo de compromisos de parte de las personas a quienes directamente va a afectar los procesos de cambio y con los que se precisa establecer formas de coordinación y retroalimentación bastante estrechas. Estas personas a las que se le puede denominar agentes o promotoras del cambio, también necesitan mecanismos motivadores que los lleve a asumir un compromiso cada vez más intenso. Finalmente, es preciso dar cabi- 
13. Tello, Max et al. "Empresa y comunicación.

Estado de la comunicación empresarial en el Perú". Cuadernos Cicosul N ${ }^{\circ} 17$. Facultad de Ciencias de la Comunicación. Lima: Universidad de Lima.

14. Rogers, Everett [y] Agarwala-Rogers, Rekha. La comunicación en las organizaciones. México: McGraw Hill, 1980, p.171. da en la propia estrategia a mecanismos que, en el breve tiempo, permitan adecuar y reformular la estrategia.

Detectados los problemas organizacionales y las brechas en el desempeño de las empresa, se debe proceder a elaborar instrumentos de diagnóstico organizacional que posibiliten cambios planificados y no cambios poco productivos como los presentados en el estudio Empresa y comunicación. Estado de la comunicación empresarial en el Perú ${ }^{13}$.

La decisión consciente para adoptar nuevas formas de hacer las cosas puede ser considerada como el primer paso en este proceso de planificación. No se debe tomar esta decisión si previamente no se ha identificado lo que se va a modificar, si no se ha precisado con lo que se le va a sustituir; no se debe improvisar en este campo. Asimismo, debemos conocer los recursos económicos y materiales con los que contamos, y el elemento humano comprometido con este proceso de cambio. Para Rogers y Agarwala la decisión empresarial de conjugar el problema, la solución (la alternativa) y los recursos son el punto de quiebre que permite señalar el momento exacto en que el cambio está presente y la necesidad de que sea incorporado ${ }^{14}$.

Una segunda fase a considerar en la planificación del cambio es la magnitud que le queremos dar, es decir, si sólo intentamos buscar una acción limitada o si queremos que el cambio involucre a toda la organización. Esta fase permitirá distribuir los recursos dados para este fin y asignar las responsabilidades a las diferentes personas comprometidas con el proceso de cambio. Estos recursos y las responsabilidades asignadas deben ser objeto de un monitoreo estrecho si queremos que los cambios tengan efectos duraderos.

Diferentes estudiosos de los grupos humanos coinciden en señalar que a mayor nivel de compromiso público de las personas con su grupo, mayor responsabilidad de realizarlo y de hacerlo con voluntad. El énfasis se orienta a la convicción más que a la imposición. Pero, igualmente se reconoce un cierto grado de procedimiento semicoactivo en los momentos iniciales del cambio con el fin de señalar la ruta por donde éste tiene que transcurrir.

Una tercera etapa se refiere a la instalación propiamente dicha del cambio empresarial. Este proceso consiste en dar el salto y relacionar el cambio buscado a nivel puntual con la estructura ya en marcha de la empresa, así como buscar los mecanismos que la engarcen con las actividades rutinarias de la empresa. No debe producirse la sensación de un caso aislado en experimentación, así lo estuviese; debe pensarse que en algún momento tiene que ser integrado al 
sistema total y no ser considerado en forma aislada, al cual se le contempla hasta estar seguro de sus resultados. Ello puede generar dudas, propiciar suspicacias y dar cabida a un ambiente más de expectativa que de apoyo. El personal prefiere ser un espectador que un comprometido con el cambio, que haría lo imposible porque éste tenga éxito y se implante en su empresa.

La última fase tiene que ver con los procedimientos que nos posibiliten institucionalizar el cambio. Esta etapa busca eliminar la condición de "novedad" del elemento que se desea incorporar a la empresa, hasta convertirlo en parte del sistema. Al término del proceso de cambio, lo nuevo ya no es identificable para la organización como una innovación; por el contrario, se convierte en parte regular de ésta y se integra al funcionamiento total. Se le incorpora por el tiempo que todo cambio en una cultura organizacional que lo promueve, debe de quedarse; luego el ciclo de detección de brechas de desempeño vuelve a iniciarse.

\section{LA COMUNICACIÓN Y EL CAMBIO ORGANIZACIONAL}

La comunicación, sin duda, es la clave que asegura el éxito en todo proceso de cambio. El compartir información sobre lo que puede identificarse colectivamente como brecha de desempeño y el establecer las alternativas frente a lo que se debe cambiar, si bien parten de un grupo restringido, luego requieren ser compartidas y aceptadas por un grupo más numeroso, que las asume como propias y las ponen en operación. Todos estos pasos más la retroalimentación permanente no serian posibles sin una comunicación fluida entre las partes comprometidas con el cambio. En consecuencia, sólo a través del entendimiento de los procesos comunicacionales en las empresas es posible comprender cabalmente la introducción de cambios y el éxito o no de ellos.

\section{La fase de detección de necesidad de cambios}

Si bien las organizaciones se mueven entre los sistemas que las circundan y existe una mutua interdependencia, la necesidad de cierta certidumbre debe igualmente estar presente. Esto lleva a los comunicadores a incorporar una racionalidad en el cambio y a elaborar instrumentos de recolección de información necesarios para un diagnóstico de necesidades tanto del fenómeno comunicacional como de otro tipo de carencias y expectativas presentes en los integrantes 
15. Thompson, James. Organizaciones en acción. Bogotá: McGraw Hill, 1994, p. 14. de las organizaciones o en el medio ambiente. Este proceso de búsqueda, señalado con claridad por James Thompson ${ }^{15}$, permite compilar la necesaria información que lleva a una racional jerarquización de necesidades y a una acertada toma de decisiones.

\section{La fase de implementación y monitoreo del cambio}

Durante los procesos de cambio organizacional, la cooperación y la cohesión son imprescindibles; la comunicación desempeña una función de primer orden. La transmisión de la información a quienes corresponda para que el cambio tenga éxito, así como el intercambio de mensajes durante las fases de cambio, tornan necesario el desarrollo de mecanismos de comunicación que garanticen un proceso sin altibajos.

\section{La fase de la realimentación}

Algunos autores la denominan la fase evaluativa, permite enmendar rumbos. Es la fase que, igualmente, posibilita la medición del impacto de los cambios. Sugiere nuevos cursos de acción y enriquece el aprendizaje acerca de lo que podemos modificar con la fuerza propia de la organización y aquéllos que requerirán de la concurrencia de variables no controlables, que son propias de los sistemas abiertos. Finalmente, posibilita cerrar el circuito en los procesos de comunicación e iniciar una nueva etapa en este permanente proceso de aprendizaje; vivir en una cultura de cambio empresaria (véase esquema anexo).

Los procesos de cambio no son uniformes en todas las organizaciones ni los períodos de duración de cada una de las fases del cambio están predeterminados; cada empresa puede trabajar a su ritmo e implementar cada etapa en el momento que se vea más oportuno. Lo que no se debe hacer es alterar las fases que presentamos. Los modelos deben seguir el proceso en todo su curso si se quiere entender los verdaderos efectos que los cambios producen. 
Dimensión proyectiva (ID)

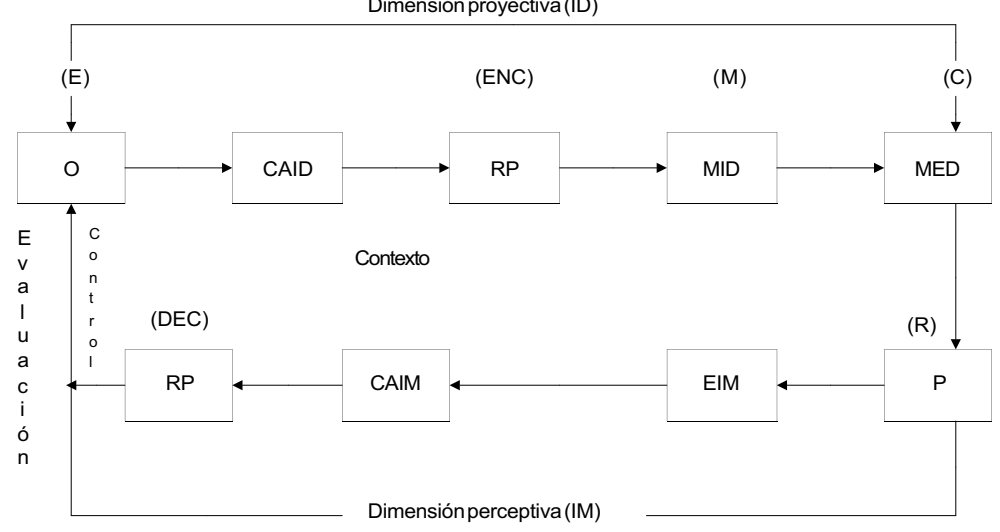


Dimensión proyectiva(ID)

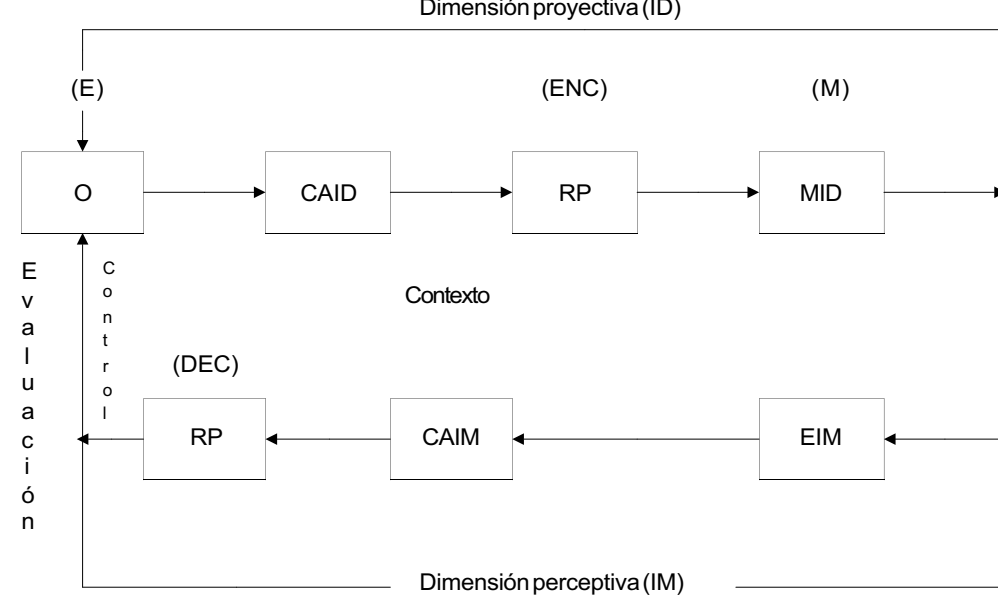


Dimensión proyectiva (ID)

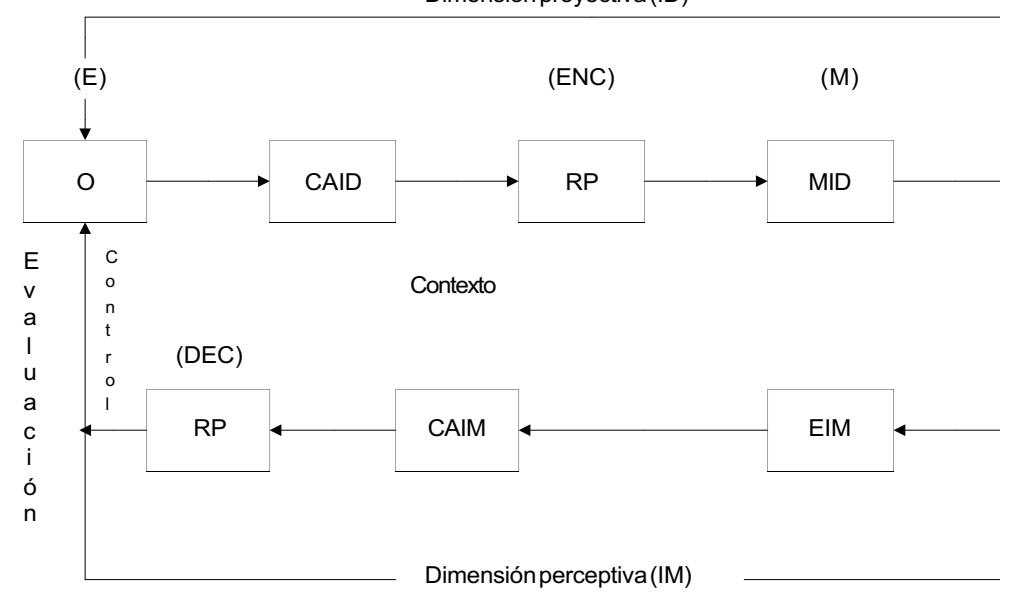


Bibliografía

Blake, Robert

1991

La estrategia para el cambio organizacional. Delaware: Addison-Wesley Iberoamericana.

Cicosul

1994

"Empresa y comunicación". Cuadernos Cicosul $\mathrm{N}^{\circ}$ 17. Facultad de Ciencias de la Comunicación. Lima: Universidad de Lima.

Hodgetts, Richard [y] Steven Altmann 1981

Comportamiento en las organizaciones. México: Editorial Interamericana.

Ogburn, William

1993

Recent Social Trends. Nueva York: McGraw Hill. 